\title{
Delayed treatment with oleanolic acid attenuates tubulointerstitial fibrosis in chronic cyclosporine nephropathy through $\mathrm{Nrf} 2 / \mathrm{HO}-1$ signaling
}

Yu Ah Hong ${ }^{1}$, Ji Hee Lim², Min Young Kim², Eun Nim Kim² ${ }^{2}$ Eun Sil Koh ${ }^{2,3}$, Seok Joon Shin ${ }^{2,4}$, Bum Soon Choi ${ }^{2,5}$, Cheol Whee Park ${ }^{2,5}$, Yoon Sik Chang ${ }^{2,3}$ and Sungjin Chung ${ }^{2,3^{*}}$

\begin{abstract}
Background: Nuclear factor erythroid-2-related factor-2 (Nrf2) is known to protect against tissue injury by orchestrating antioxidant and detoxification responses to oxidative stress. This study investigated whether upregulation of Nrf2-dependent signaling by oleanolic acid (OA), which is known to activate Nrf2, could attenuate renal inflammation and fibrosis in cyclosporine (CSA)-induced kidney injury.

Methods: Male ICR mice were divided into four treatment groups: Vehicle $(\mathrm{VH}, \mathrm{n}=6), \mathrm{VH}+\mathrm{OA}(\mathrm{n}=6), \mathrm{CsA}(\mathrm{n}=8)$, and $\mathrm{CsA}+\mathrm{OA}(\mathrm{n}=8)$. For the OA-treated groups, OA $(25 \mathrm{mg} / \mathrm{kg} /$ day) was administered by intraperitoneal injection for the final week of the 4-week experimental period. Renal function, morphologies and signaling were evaluated at the end of the study.

Results: Treatment with CSA resulted in decreased kidney function and urine osmolality and increased urine volume and urinary albumin levels. The CsA-induced changes were improved by OA treatment. Specifically, administration of OA decreased tubulointerstitial fibrosis and inflammation scores that were increased in CsA-treated mice. Furthermore, OA treatment decreased urinary 8-hydroxy-2'-deoxyguanosine (8-OHdG) and 8-epi-prostaglandin F2a (8-iso-PGF2a) levels. The beneficial effects of OA were attributed to an increased ratio of nuclear/total Nrf2 and subsequently enhanced expression of heme oxygenase (HO)-1, as well as a stable level of Kelch-like ECH-associated protein 1 (Keap1) expression, indicating that $\mathrm{OA}$ enhanced nuclear translocation of Nrf2. Increased apoptotic cell death and a high ratio of B cell leukaemia/lymphoma 2 (Bcl-2)-associated X protein (Bax) to Bcl-2 in CsA-treated mice were also significantly ameliorated by OA treatment.
\end{abstract}

Conclusion: Our results suggest that OA activates Nrf2/HO-1 signaling in chronic CsA nephropathy, which may have beneficial effects on inflammation and oxidative stress.

Keywords: Cyclosporine, Kidney, Fibrosis, Oleanolic acid, Oxidative stress

\section{Background}

Cyclosporine A (CsA) is one of the most effective and widely used immunosuppressants in solid organ transplantation and autoimmune disease. Despite the beneficial effects of CsA, its use in the clinic is limited by its nephrotoxic potential. CsA-induced nephropathy is characterized by progressive renal insufficiency, arteriopathy of

\footnotetext{
* Correspondence: sungjin.chung@outlook.com

2Department of Internal Medicine, College of Medicine, The Catholic University of Korea, 222 Banpo-daero, Seoul 137-701, Republic of Korea ${ }^{3}$ Division of Nephrology, The Catholic University of Korea Yeouido St. Mary's Hospital, 10, 63-ro, Yeongdeungpo-gu, Seoul 150-713, Republic of Korea Full list of author information is available at the end of the article
}

the afferent arterioles, tubulointerstitial inflammation, and striped fibrosis [1]. Although the exact mechanism of CsA-induced nephrotoxicity remains obscure, oxidative stress and apoptotic cell death can play a pivotal role in producing structural and functional kidney impairment in CsA-induced renal injury [2,3]. Therefore, it is necessary to control inflammation, apoptosis, and fibrosis associated with oxidative stress in order to delay progression of chronic CsA nephropathy.

Studies on the renal protective response and pathways responsible for activation of intracellular specific signaling molecules or genes should aid in our understanding 
of oxidative stress and development of future therapeutics. Recently, nuclear factor E2-related factor 2 (Nrf2) was found to be a critical transcription factor that binds to antioxidant response elements (AREs) present in the promoter region of a number of genes encoding antioxidant and phase 2 enzymes, including glutathione peroxidase, catalase, and superoxide dismutase (SOD) [4,5]. Nrf2 appears to function upon being released from its repressor Kelchlike ECH-associated protein 1 (Keap1) by sensing cytoplasmic oxidative stress or other specific chemical agents [6,7]. Heme oxygenase (HO)-1, an important Nrf2 target gene, catalyzes heme metabolism, yielding iron, carbon monoxide and bilirubin. Likewise, HO-1 is recognized as a protective gene in the kidney involved in degradation of pro-oxidant heme, resulting in production of anti-inflammatory, antioxidant, and anti-apoptotic metabolites [8].

The cytoprotective effects of Nrf2 are supported by previous studies showing that Nrf2 gene ablation intensifies inflammation, oxidative stress and histological changes $[9,10]$. Oleanolic acid (OA), a known activator of Nrf2, is a natural pentacyclic triterpenoid that has hepatoprotective, anti-inflammatory, antioxidant, and anticancer activities [11]. Recently, OA has been reported to have renoprotective effects in experimental animals using toxicants and streptozocin $[12,13]$. However, there have been no studies on the renoprotective effects of OA in a mouse model of chronic CsA nephropathy. Thus, the aim of this study was to investigate whether upregulation of Nrf2-depedent antioxidative signaling by OA could attenuate renal oxidative stress and fibrosis in CsA-induced nephropathy. In addition, we delayed administration of OA to determine whether Nrf2 activated by $\mathrm{OA}$ treatment confers renoprotective effects when initiated after the onset of CsA-induced damage.

\section{Materials and methods Experimental design}

Five-week-old male ICR mice (DBL, Chungcheongbuk-do, Republic of Korea), initially weighing 15 to $20 \mathrm{~g}$, were housed in a temperature- and light-controlled environment and allowed free access to a low-salt diet $(0.01 \%$ sodium; Research Diets, NJ, USA) and tap water. Mice were randomized into four subgroups and treated daily for 28 days: (1) Vehicle-treated control group $(\mathrm{VH}, \mathrm{n}=6)$ was given a daily subcutaneous injection of vehicle (olive oil $1 \mathrm{~mL} / \mathrm{kg}$, Sigma-Aldrich, St. Louis, MO, USA); (2) Vehicle plus OAtreated group $(\mathrm{VH}+\mathrm{OA}, \mathrm{n}=6)$ was given a daily subcutaneous injection of olive oil $(1 \mathrm{~mL} / \mathrm{kg})$, and in this group, OA (25 mg/kg; Sigma-Aldrich, St. Louis, MO, USA) was injected intraperitoneally daily for one week; (3) CsA onlytreated group (CsA, $\mathrm{n}=8$ ) was given a daily subcutaneous injection of CsA (30 mg/kg; Chong Kun Dang, Seoul, Republic of Korea); and (4), the CsA plus OA-treated group $(\mathrm{CsA}+\mathrm{OA}, \mathrm{n}=8)$ was given a daily subcutaneous injection of CsA, and OA was given by daily intraperitoneal injection for the final week of the experimental period. The doses of CsA $[14,15]$ and OA [16] were selected based on previous reports. The experimental protocol was approved by the Institutional Animal Care and Use Committee of The Catholic University of Korea (CUMC-2012-0093-02).

\section{Measurement of basic parameters}

Before sacrifice, animals were housed individually in metabolic cages (Nalge, Rochester, NY, USA) for 24-hr urine collection. Albuminuria and urine creatinine were measured using ELISA kits (Exocell, Philadelphia, PA, USA). Measurement of serum creatinine concentrations and urine osmolality was performed at Samkwang Medical Laboratories (Seoul, Korea) using enzymatic colorimetric methods (Modular DPP system, Roche, Hamburg, Germany). Creatinine clearance was calculated using a standard formula (urine creatinine $(\mathrm{mg} / \mathrm{dL}) \times$ urine volume $(\mathrm{mL} / 24 \mathrm{~h}) /$ serum creatinine $(\mathrm{mg} / \mathrm{dL}) \times 1440(\mathrm{~min} / 24 \mathrm{~h}))$. Whole-blood CsA concentrations were quantified using liquid chromatography-tandem mass spectrometry with an API3200 LC-MS System (Applied Biosystems/MDS Sciex, Foster City, CA, USA) equipped with an electrospray ionization interface to generate negative ions $[\mathrm{M}+\mathrm{NH} 4]^{+}$.

\section{Histopathology}

On the day of sacrifice, kidneys were retrieved, washed with heparinized saline, fixed in a periodate-lysineparaformaldehyde solution, and embedded in wax. After dewaxing, $4 \mu \mathrm{m}$ sections were processed and stained with hematoxylin-eosin (H\&E), periodic acid Schiff (PAS), and Masson's trichrome. Tubulointerstitial fibrosis (TIF) was defined as a matrix-rich expansion of the interstitium with tubular dilatation, atrophy, cast formation, and sloughing of tubular epithelial cells or thickening of the tubular basement membrane. At least 20 fields per section were assessed by counting the percentage of injured areas per field of cortex at 200× magnification with a color-image analyzer (TDI Scope Eye Version 3.5 for Windows; Olympus, Japan).

\section{In situ TUNEL assay}

Apoptosis was assessed using terminal deoxynucleotidyl transferase-mediated biotin nick end-labeling (TUNEL) assay. Detection of apoptotic cells in formalin-fixed, paraffin-embedded tissue was performed by in situ TUNEL using the ApopTag In Situ Apoptosis Detection Kit (Chemicon-Millipore, Billerica, MA, USA) according to the manufacturer's protocol. TUNEL positive cells were evaluated at $400 \times$ magnification.

\section{Assessment of markers for renal oxidative stress}

Twenty-four-hour urinary concentrations of 8-hydroxy2'-deoxyguanosine (8-OHdG; OXIS Health Products, 
Table 1 Biochemical and physical characteristics of the four groups at the end of the 4-week period

\begin{tabular}{lcccc}
\hline & VH $(\mathbf{n}=\mathbf{6})$ & VH + OA $(\mathbf{n}=\mathbf{6})$ & CsA ( $\mathbf{n}=\mathbf{6})$ & CsA + OA (n=8) \\
\hline Body weight $(\mathrm{g})$ & $35.77 \pm 5.25$ & $34.3 \pm 1.45$ & $33.97 \pm 2.34$ & $32.78 \pm 4.46$ \\
Kidney weight $(\mathrm{g})$ & $0.23 \pm 0.04$ & $0.23 \pm 0.02$ & $0.18 \pm 0.04$ & $0.22 \pm 0.03$ \\
Urine volume $(\mathrm{mL})$ & $1.53 \pm 0.97$ & $2.53 \pm 1.71$ & $7.15 \pm 5.15^{\mathrm{b}}$ & $1.4 \pm 1.96$ \\
Serum Cr $(\mathrm{mg} / \mathrm{dl})$ & $0.21 \pm 0.02$ & $0.26 \pm 0.02$ & $0.30 \pm 0.11$ & $0.25 \pm 0.06$ \\
Cr Clearance $(\mathrm{mL} / \mathrm{min} / 100 \mathrm{~g} \mathrm{BW})$ & $0.72 \pm 0.15$ & $0.44 \pm 0.09$ & $0.26 \pm 0.16^{\mathrm{a}}$ & $0.52 \pm 0.19$ \\
24 hr albuminuria $(\mu \mathrm{g} / \mathrm{day})$ & $23.90 \pm 4.48$ & $26.75 \pm 11.88$ & $150.99 \pm 57.42^{\mathrm{a}}$ & $57.34 \pm 7.44$ \\
Urine osmolality $(\mathrm{mOsm} / \mathrm{kg})$ & $2088.67 \pm 963.44$ & $810.67 \pm 181.88$ & $582.00 \pm 208.89^{\mathrm{b}}$ & $2143.33 \pm 547.01$ \\
CsA concentration $(\mathrm{ng} / \mathrm{mL})$ & $\mathrm{N} / \mathrm{A}$ & $\mathrm{N} / \mathrm{A}$ & $1792.34 \pm 63.08$ & $1764.40 \pm 28.56$ \\
\hline
\end{tabular}

Data are means \pm SEM. ${ }^{\mathrm{a}} \mathrm{p}<0.05$ compared with vehicle, ${ }^{\mathrm{b}} \mathrm{p}<0.05$ compared with other groups. $\mathrm{VH}=$ vehicle-treated group; $\mathrm{VH}+\mathrm{OA}=\mathrm{Vehicle}$ plus oleanolic acidtreated group; $\mathrm{CsA}=$ cyclosporine-treated group; $\mathrm{Cs} A+\mathrm{OA}=$ cyclosporine plus oleanolic acid-treated group; $\mathrm{Cr}=\mathrm{creatinine} ; \mathrm{BW}=$ body weight; $\mathrm{N} / \mathrm{A}=\mathrm{not}$ available.

OR, USA) and 8-epi-prostaglandin F2 $\alpha$ (8-epi-PGF2 $\alpha$; OxisResearch, CA, USA) were measured using a competitive enzyme-linked immunosorbent assay according to the manufacturer's protocol. Lipid peroxidation as an index of oxidative stress was determined by assaying malondialdheyde (MDA) production with the thiobar- bituric Acid Reactive Substance (TBARS) test (OxiSelect MDA Adduct ELISA Kit, Cell Biolabs Inc., CA, USA).

Immunohistochemistry for cleaved caspase-3 and a-SMA Presence of cleaved caspase- 3 and $\alpha$-smooth muscle actin ( $\alpha$-SMA) was determined by immunohistochemistry.

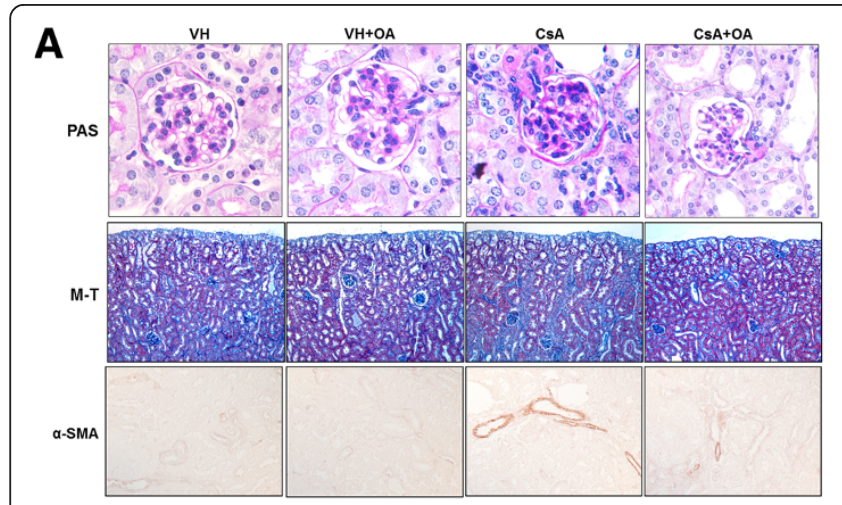

\section{Tubulointerstitial fibrosis (folds)}

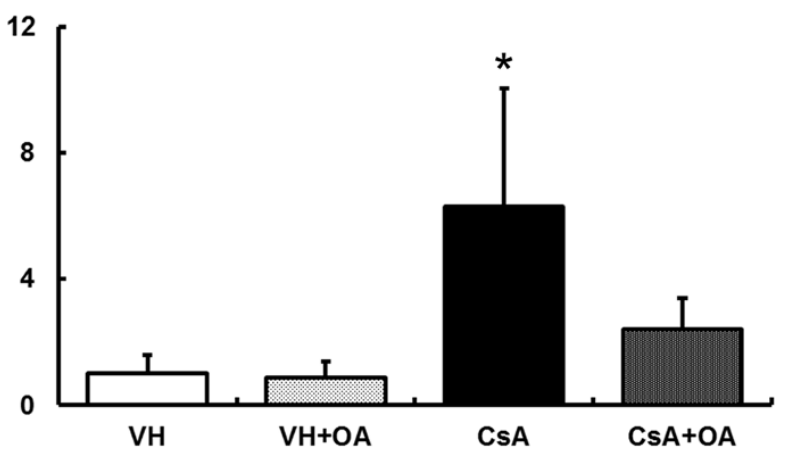

\section{B Fractional Mesangial Area (\%)}
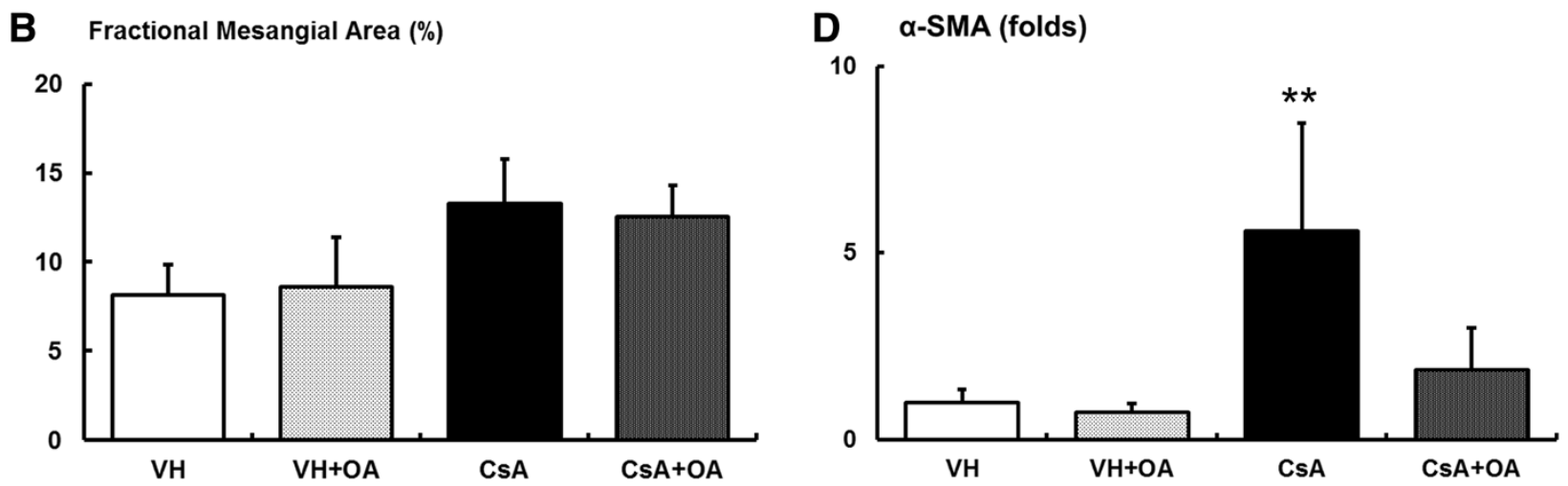

Figure 1 Effects of oleanolic acid (OA) on renal morphological changes. (A) Representative renal sections stained with periodic acid-Schiff (PAS; original magnifications, $\times 400$ ), Masson-trichrome (M-T; original magnifications, $\times 400)$, and $a-$-smooth muscle actin (a-SMA; original magnifications, $\times 200$ ). (B) Quantitative analyses of the results for the mesangial fractional area (\%). (C) Quantitative analyses of the results for the tubulointerstitial fibrosis area. ${ }^{*} p<0.01$ vs. other groups. (D) Quantitative analyses of the results for $a-S M A .{ }^{*} p<0.05$ vs. other groups. $\mathrm{VH}=$ vehicle-treated group; $\mathrm{VH}+\mathrm{OA}=$ vehicle plus oleanolic acid-treated group; $\mathrm{CsA}=$ cyclosporine-treated group; $\mathrm{CsA}+\mathrm{OA}=$ cyclosporine plus oleanolic acid-treated group. 
Briefly, small blocks of kidney were immediately fixed in $10 \%$ buffered formalin for $24 \mathrm{~h}$ before being embedded in paraffin. Next, $4 \mu \mathrm{M}$ thick sections of renal tissues were incubated overnight with anti-cleaved caspase-3 (1:100; Abcam, Cambridge, UK) or anti- $\alpha$-SMA (1:500; Abcam, Cambridge, UK) in a humidified chamber at $4^{\circ} \mathrm{C}$. The primary antibodies were localized with a peroxidaseconjugated secondary antibody and developed using the Vector Impress kit (Vector Laboratories, Burlingame, CA, USA) and 3, 3-diamninobenzidine substrate solution with nickel chloride enhancement. Sections were then dehydrated in ethanol, cleared in xylene, and mounted without

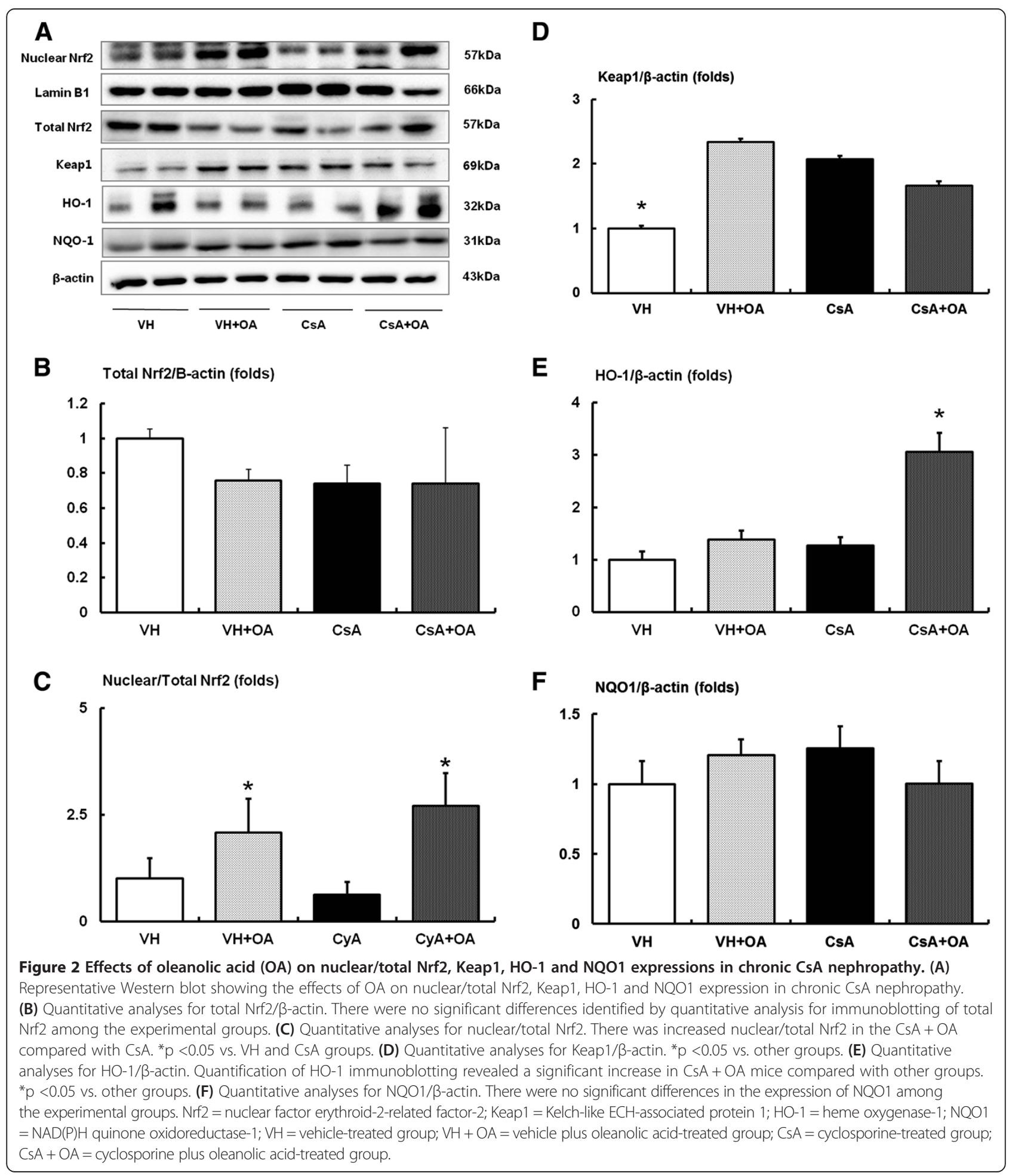


counterstaining. All of these sections were examined in a blinded manner using light microscopy (Olympus BX50, Olympus Optical, Tokyo, Japan). For the quantification of the proportional areas of staining, approximately 20 views (400× magnifications) were randomly located in the renal cortex and the corticomedullary junction of each slide.

\section{Western blotting}

For Western blot analysis, total protein of renal cortical tissues was extracted with a Pro-Prep Protein Extraction Solution (Intron Biotechnology, Gyeonggi-do, Korea)

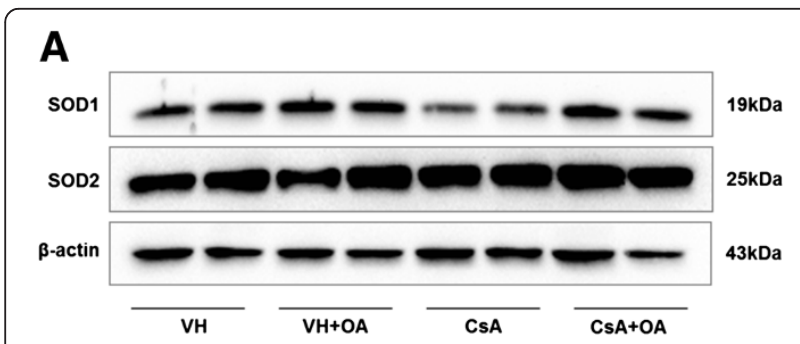

B SOD1/ $\beta$-actin (folds)

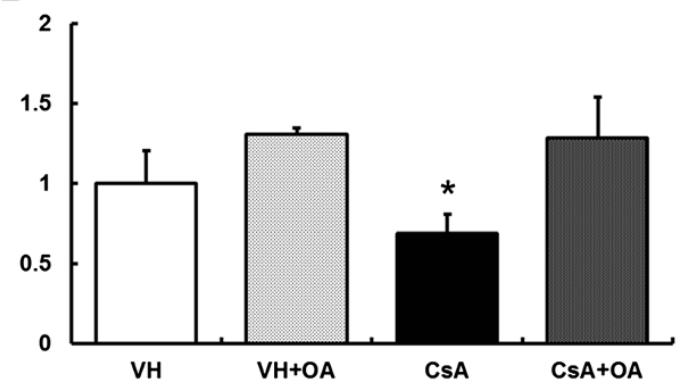

\section{SOD2/ $\beta$-actin (folds)}

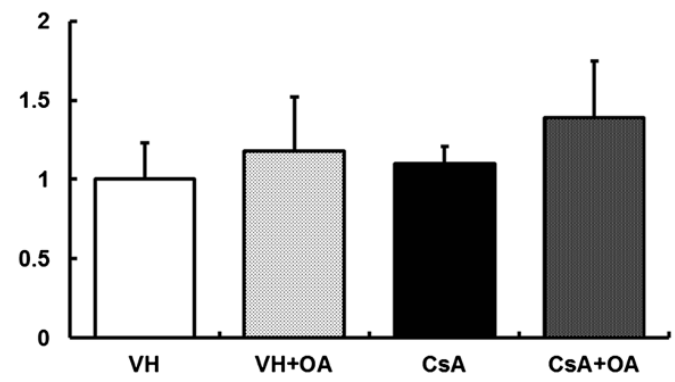

Figure 3 Effect of oleanolic acid (OA) on expressions of SODs in chronic CsA nephropathy. (A) Immunoblotting for the target molecules of SOD1 and SOD2 showing the effects of OA in chronic CSA nephropathy. (B) Quantitative analyses for SOD1/ $\beta$-actin. The expression of SOD1 was significantly decreased in CSA but increased in CsA + OA mice. * $p<0.05$ vs. other groups. (C) Quantitative analyses for SOD2/ $\beta$-actin. There were no significant differences in the expression of SOD2 among the experimental groups. SOD = superoxide dismutase; $\mathrm{VH}=$ vehicle-treated group; $\mathrm{VH}+\mathrm{OA}=$ vehicle plus oleanolic acid-treated group; $\mathrm{Cs} A=$ cyclosporine-treated group; $\mathrm{Cs} \mathrm{A}+\mathrm{OA}=$ cyclosporine plus oleanolic acid-treated group. according to the manufacturer's instructions. Nuclear extracts, which were used for Nrf2 immunoblotting, were prepared with the NE-PER nuclear kit as previously described (Pierce Biotechnology, Rockford, IL) [17]. Western blotting was performed for nuclear Nrf2, total Nrf2, Keap1, HO-1, NAD(P)H quinone oxidoreductase-1 (NQO1), B cell leukaemia/lymphoma 2 (Bcl-2), Bcl-2associated $\mathrm{X}$ protein (Bax), SOD1, SOD2, and catalase. Specifically, proteins were separated by SDS-PAGE, transferred to nitrocellulose membranes, and detected with the following antibody concentrations: Nrf2 (1:1000; Santa Cruz Biotechnology Inc, Texas, USA), Keap1 (1:1000; Santa Cruz Biotechnology Inc, Texas, USA), HO-1 (1:1000; BD Biosciences, California, USA), NQO1 (1:1000; Santa Cruz Biotechnology Inc, Texas, USA), Bcl-2 (1:500; Santa Cruz Biotechnology Inc, Texas, USA), Bax (1:500; Santa Cruz Biotechnology Inc, Texas, USA), SOD1 (1:5000; Assay Designs, MI, USA), SOD2 (1:10000; Abcam, Cambridge, UK), Catalase (1:2000; Abcam, Cambridge, UK), and $\beta$-actin (1:10000; Sigma-Aldrich, MO, USA).

\section{Statistical analysis}

Data are expressed as the mean \pm SEM. Multiple comparisons between groups were performed by one-way analysis of variance with post hoc tests. Statistical significance was assumed as $\mathrm{p}<0.05$.

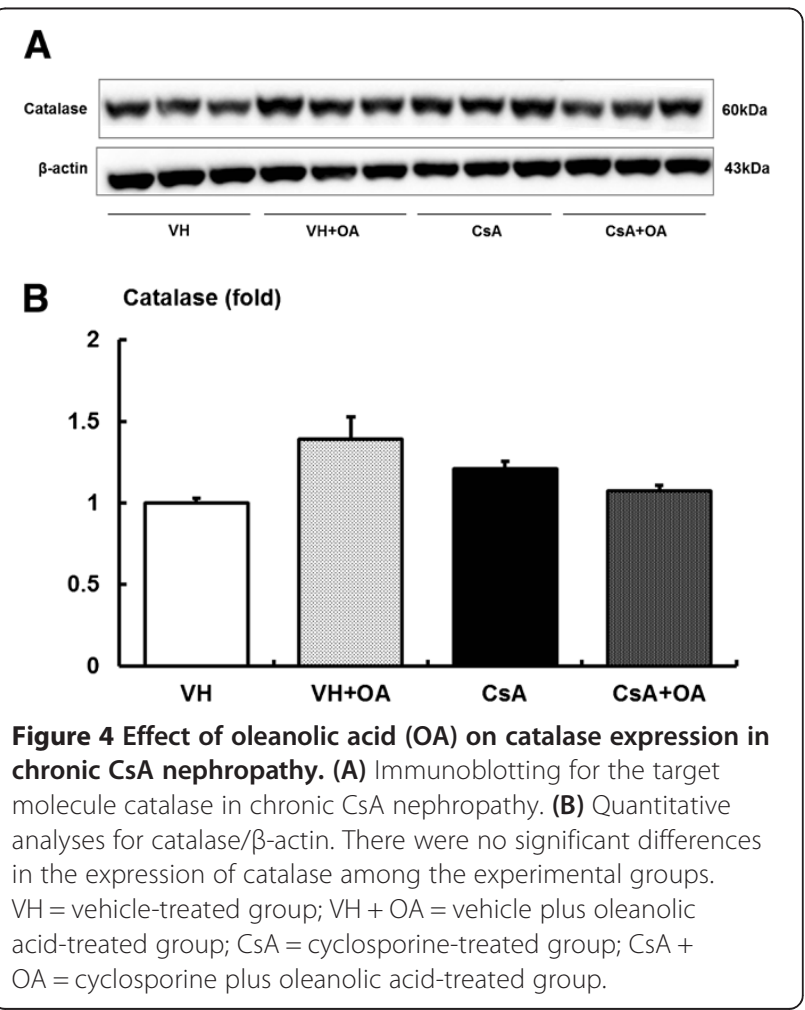




\section{Lipid peroxidation (nmol MDA/mg protein)}

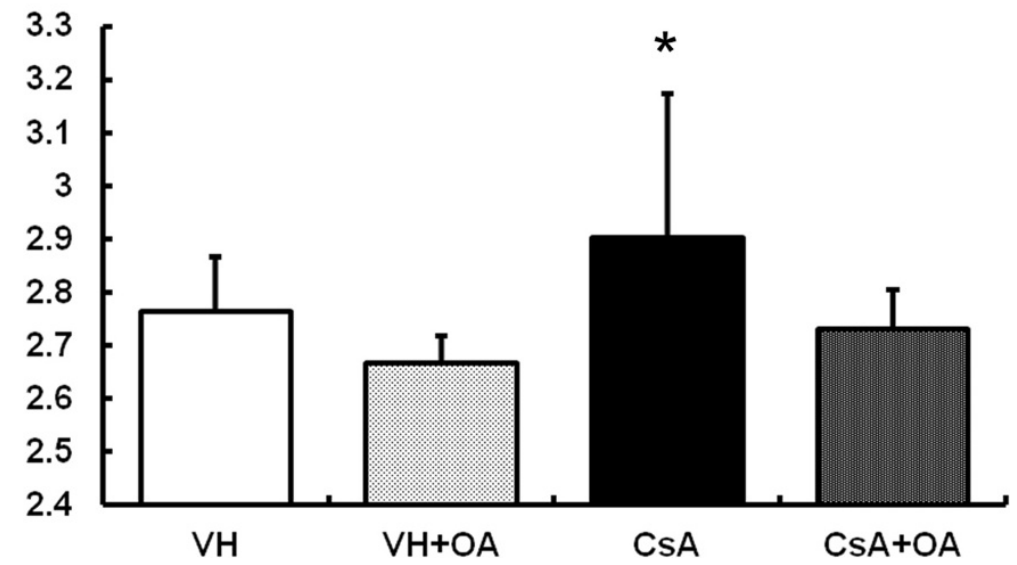

Figure 5 Assay for concentrations of the lipid peroxidation marker malonedialdehyde (MDA) in renal cortical tissue among the experimental groups. ${ }^{*} \mathrm{p}<0.05$ vs. other groups. $\mathrm{VH}=$ vehicle-treated group; $\mathrm{VH}+\mathrm{OA}=$ vehicle plus oleanolic acid-treated group; $\mathrm{CsA}=\mathrm{cyclosporine-}$ treated group; $\mathrm{Cs} A+\mathrm{OA}=$ cyclosporine plus oleanolic acid-treated group.

\section{Results}

\section{Renal functional parameters}

Table 1 shows the changes in functional parameters among all groups at the end of the 4-week study period. Compared with $\mathrm{VH}$ and $\mathrm{VH}+\mathrm{OA}$, the CsA-treated groups $\mathrm{CsA}$ and $\mathrm{CsA}+\mathrm{OA}$ had a slightly reduced body weight, although the difference was not statistically significant. Urine volume and 24-hour albuminuria were significantly increased in the CsA group compared with the $\mathrm{VH}$ and $\mathrm{VH}+\mathrm{OA}$ groups. OA treatment improved albuminuria and decreased urine volume to the level of the control groups $\mathrm{VH}$ and $\mathrm{VH}+\mathrm{OA}$. There was a significant decrease in urine osmolality in CsA mice compared with those in the $\mathrm{VH}$, and a significant increase in $\mathrm{CsA}+\mathrm{OA}$ group. We also observed decreased creatinine clearance in mice given CsA compared to mice in the $\mathrm{VH}$ group. However, administration of OA inhibited the decline in creatinine clearance in CsA-treated mice.

\section{Effects of oleanolic acid on renal morphological changes}

There was no significant difference of fractional mesangial area among all study groups (Figure $1 \mathrm{~A}$ and $1 \mathrm{~B}$ ). TIF was produced in the cortex of the kidney of CsAtreated mice. Quantitative analysis showed that renal fibrosis was significantly increased in mice given CsA compared with $\mathrm{VH}$ and $\mathrm{VH}+\mathrm{OA}$ mice. By contrast, OA treatment significantly decreased TIF in CsA-treated mice (Figure $1 \mathrm{~A}$ and $1 \mathrm{C}$ ). Administration of OA significantly inhibited the CsA-induced increase in $\alpha$-SMA expression (Figure 1A and 1D).

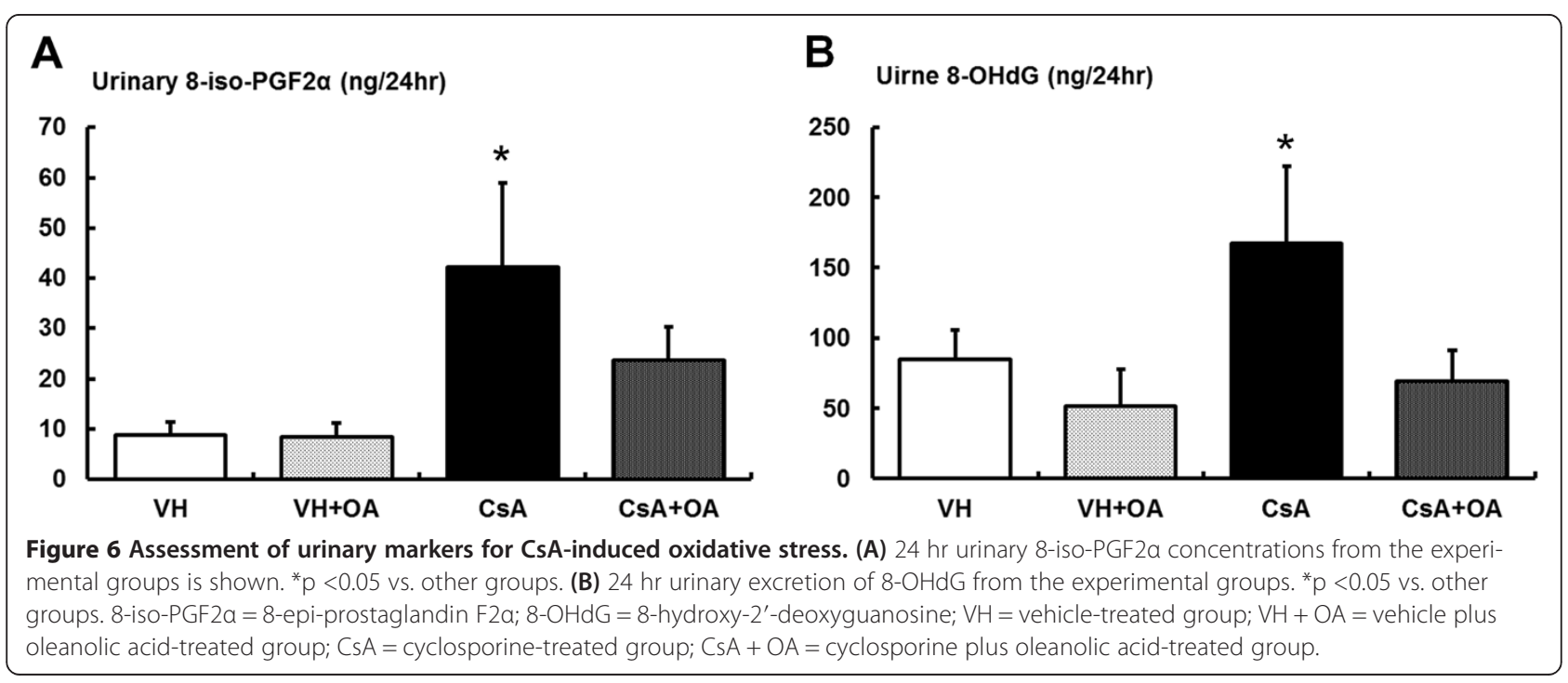


Effects of oleanolic acid on renal expression of Nrf-2, Keap1, and the antioxidant defense system

We next evaluated the effect of OA on the Nrf2/Keap1 signaling pathway (Figure 2A). The expressions of total $\mathrm{Nrf2}$ in the kidney were mildly decreased in $\mathrm{VH}+\mathrm{OA}$, $\mathrm{CsA}$ and $\mathrm{CsA}+\mathrm{OA}$ groups, but there was no significant difference between those with or without OA treatment (Figure 2B). On closer inspection, administration with OA markedly increased the level of nuclear Nrf2 in the kidneys of mice. Consequently, the intra-renal nuclear $\mathrm{Nrf} 2$ /total Nrf2 ratio was significantly increased in the $\mathrm{CsA}+$ OA group compared with the CsA group (Figure 2C). The expression of the Nrf2 repressor Keap1 significantly increased in the $\mathrm{VH}+\mathrm{OA}, \mathrm{CsA}$ and $\mathrm{CsA}+\mathrm{OA}$ groups compared with $\mathrm{VH}$ group (Figure 2D). Next, we measured the expression of two Nrf2 target proteins, NQO1 and HO-1. Administration of $\mathrm{OA}$ in CsA-treated mice resulted in a significant increase in HO-1 expression compared with the $\mathrm{VH}$ and CsA groups (Figure 2E). Conversely, there was no statistically significant difference in NQO1 levels among any of the groups (Figure 2F).

The protein levels of SOD1 and SOD2 were also evaluated (Figure 3A). SOD1 levels were significantly lower in the CsA group compared with $\mathrm{VH}$ and $\mathrm{VH}+\mathrm{OA}$ groups. In CsA-treated mice, administration of OA restored levels of SOD1 to those found in the $\mathrm{VH}$ and $\mathrm{VH}+\mathrm{OA}$ groups (Figure 3B). However, the expression of SOD2 was not different among groups (Figure 3C). Similarly, OA had no effect on catalase levels (Figure 4).

We also observed increased levels of MDA, a stable indicator of oxidative stress, in CsA-treated mice; however, administration of $\mathrm{OA}$ reversed the elevation of MDA (Figure 5). In addition, the levels of 24-hour urinary 8 -iso-PGF $2 \alpha$ and 8 -OHdG were higher in the CsA compared with control groups, and were attenuated by treatment with OA (Figure 6).

\section{Effects of oleanolic acid on renal apoptosis}

CsA treatment suppressed the expression of antiapoptotic marker $\mathrm{Bcl}-2$ and increased the expression of pro-apoptotic markers Bax and cleaved caspase- 3 (Figures 7 and 8). There was a significant increase in the $\mathrm{Bax} / \mathrm{Bcl}-2$ protein ratio for the $\mathrm{CsA}$ group compared with the $\mathrm{VH}$ and $\mathrm{VH}+\mathrm{OA}$ groups. However, the elevated $\mathrm{Bax} / \mathrm{Bcl}-2$ ratio was significantly attenuated in the $\mathrm{CsA}+\mathrm{OA}$ group (Figure 7). Furthermore, the number of TUNEL-positive cells and the expression of cleaved caspase-3 were significantly higher in the CsA group compared with the $\mathrm{VH}$ and $\mathrm{VH}+\mathrm{OA}$ groups (Figure 8A). However, administration of OA to CsA-treated mice significantly reduced the number of TUNEL-positive cells by approximately $80 \%$ compared with the CsA group without $\mathrm{OA}$ (Figure $8 \mathrm{~B}$ ). In addition, $\mathrm{OA}$ attenuated the activation of cleaved caspase-3 (Figure 8C).

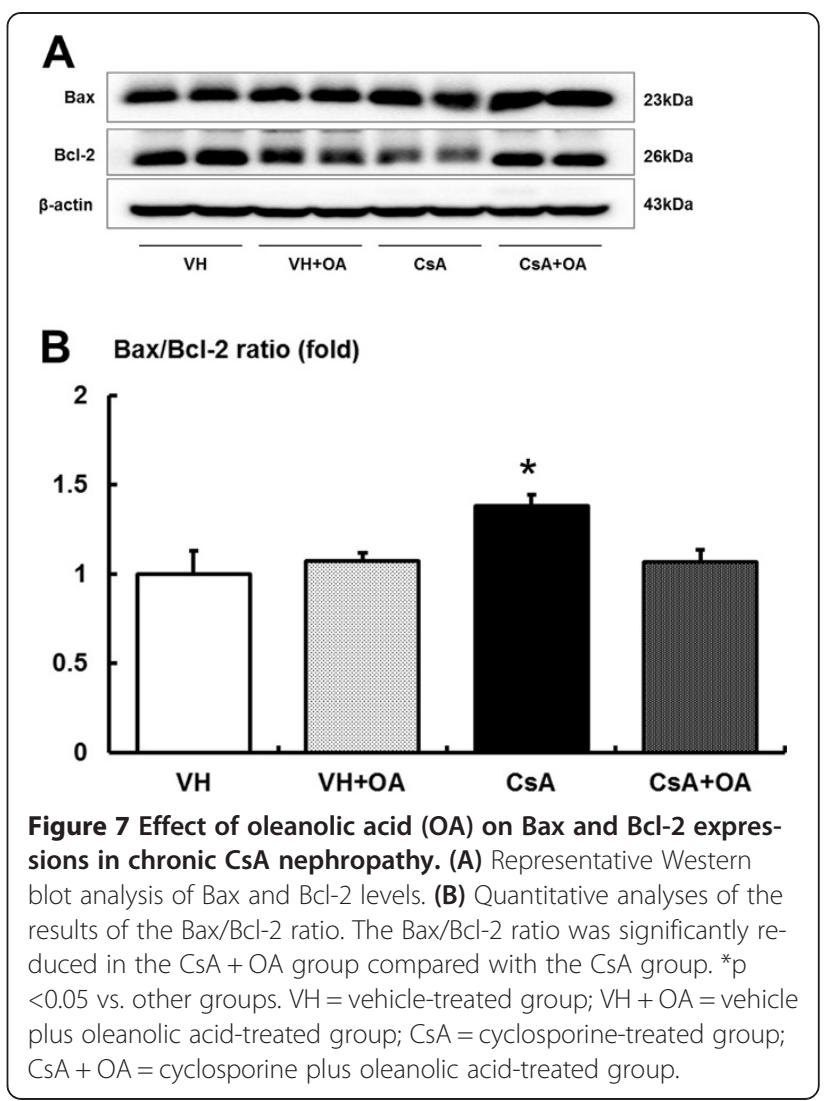

\section{Discussion}

The current study demonstrated that OA treatment ameliorated both renal dysfunction and histopathology in chronic CsA-induced nephropathy. The renoprotective mechanism of OA towards CsA-induced renal injury appeared to be associated with improvement of interstitial inflammation, fibrosis, and apoptotic cell death, as well as attenuation of oxidant stress through association with enhanced nuclear translocation of Nrf2 and subsequent activation of downstream antioxidant enzyme targets.

Numerous studies have demonstrated the relationship between pathogenesis and progression of renal diseases and oxidative stress [18-21]. CsA treatment is known to induce oxidative stress injury by increasing the production of reactive oxygen species (ROS) and MDA and decreasing the activities of antioxidant enzymes such as SOD and glutathione peroxidase [22,23]. Considering the findings that impairment of Nrf2 activity and consequent down-regulation of its antioxidant and detoxifying target genes products play a major role in the pathogenesis and progression of kidney disease [24,25], pharmacological activation of Nrf2 may be useful for treating CsA-induced renal injuries.

$\mathrm{OA}$ is widely distributed in the plant kingdom as a free acid or an aglycone of tripenoid saponins [26]. OA can be easily obtained in high yield from olive pulp 

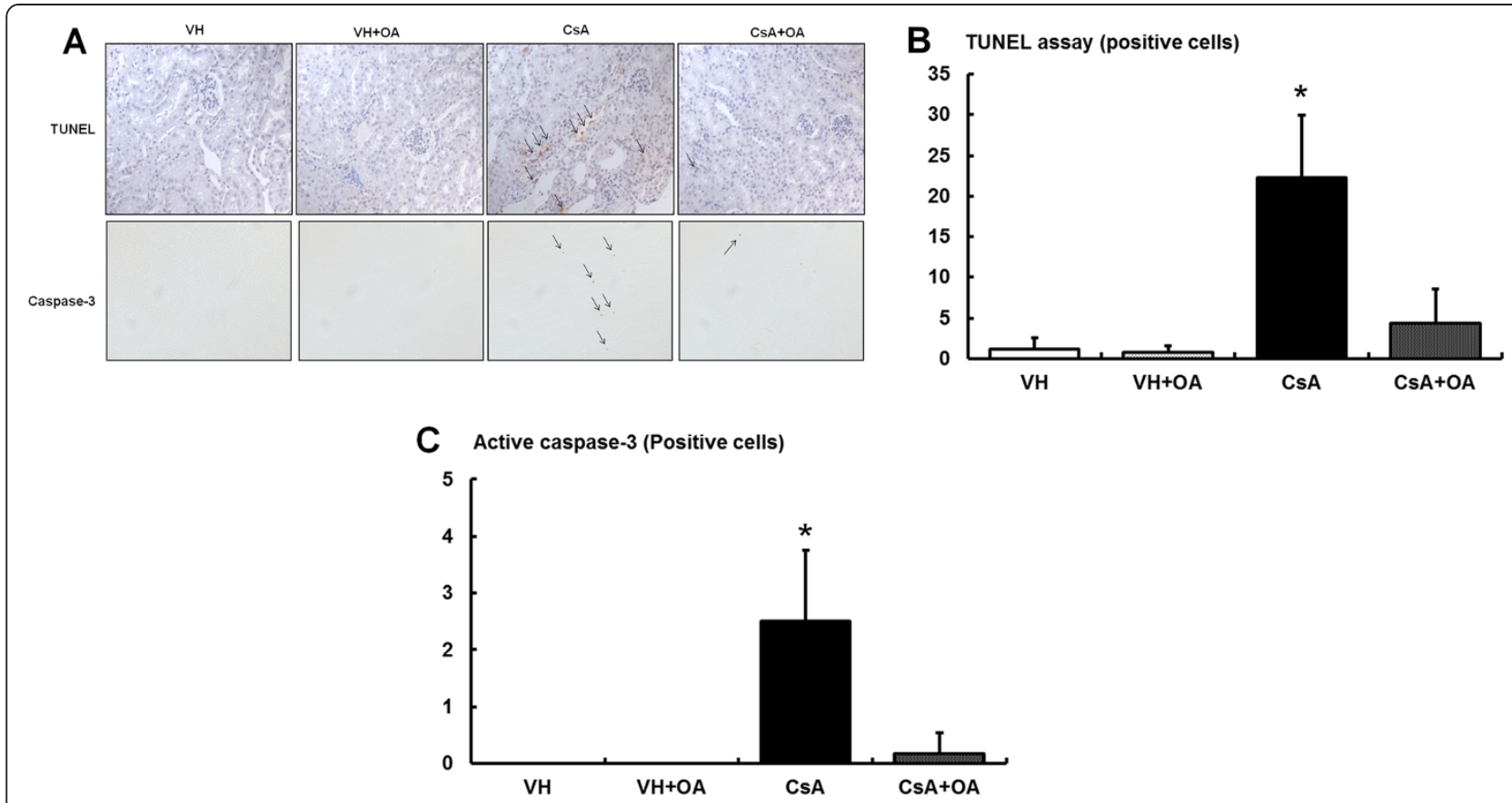

Figure 8 Effect of oleanolic acid (OA) on apoptosis in chronic CsA nephropathy. (A) TUNEL assay and immunohistochemical stain of active caspase-3 in kidney tissues of the experimental groups. (B) Quantitative analysis of TUNEL-positive nuclei in the experimental groups. The number of TUNEL-positive nuclei was significantly reduced in the CsA + OA group compared with the CsA group. * $<0.01$ vs. other groups. (C) Quantitative analysis of the cleaved caspase-3 results. Cleaved caspase-3, a pro-apoptotic marker, was significantly decreased in CsA + OA compared with CSA. ${ }^{*} p<0.01$ vs. other groups. TUNEL = terminal deoxynucleotidyl transferase-mediated biotin nick end-labeling; VH = vehicle-treated group; VH $+\mathrm{OA}=$ Vehicle plus oleanolic acid-treated group; CsA = cyclosporine-treated group; CsA + OA = cyclosporine plus oleanolic acid-treated group.

remaining after the oil is pressed from the olive fruit, as well as from olive leaves that are usually discarded after trees are pruned [27]. Previous studies have shown that OA has potent antioxidant activity and may protect several types of cells and tissues against oxidative stress [28]. The antioxidant effects of OA appear to be mediated to a great extent through activation of Nrf2 $[16,17]$. Nrf2 is a basic leucine zipper transcription factor that regulates many genes encoding detoxifying and antioxidant enzymes, including NQO1, GSH S-transferase, HO-1, glutamate cysteine ligase, peroxiredoxin, and GSH peroxidase, all of which contribute to cellular protection by removing ROS including superoxide anions, hydrogen peroxide, and hydroxyl radicals [29]. The inactive form of Nrf2 is localized in the cytoplasm bound to Keap1, a cytoskeleton-associated protein. Nrf2 activation appears to occur by various mechanisms including release of Nrf2 from Keap1, downregulation of Keap1 expression, disruption of the Keap1-Cullin-3 complex, and nuclear translocation of Nrf2 [30]. Upon exposure to oxidative stress, Nrf2 dissociates from Keap1 and translocates into the nucleus where it dimerizes with small Maf binding protein [31]. In our study, we found that administration of OA in CsA-treated mice did not result in an increase in Keap1 expression, but did increase the ratio of nuclear/total Nrf2 in CsA-treated kidneys. Although Nrf2 activation is an initial cellular adaptive response that occurs as soon as cells are challenged by oxidative stress [31], prolonged oxidative stress and inflammation appears to disturb nuclear translocation of Nrf2 in chronic CsA nephropathy. Based on the findings of our study, OA may be considered as a facilitator of nuclear translocation of cytoplasmic Nrf2 released from Keap1 following oxidative stress resulting from CsA treatment.

After translocating to the nucleus, Nrf2 is involved with protection of the cell against oxidative stress through ARE-mediated induction of several phase 2 detoxifying and antioxidant enzymes such as $\mathrm{HO}-1[32,33]$. HO-1 is expressed at low levels within normal kidneys and is induced in response to tubulointerstitial injury. In proteinuric human kidney disease, $\mathrm{HO}-1$ protein is induced in tubular epithelial cells, more prominently in distal tubules than proximal tubules, but is not expressed in resident glomerular cells. However, expression of HO-1 protein in proximal tubules, but not in distal tubules, correlates with proteinuria, hematuria, and tubulointerstitial disease $[34,35]$. A previous study suggested that downregulation of $\mathrm{HO}-1$ by CsA may be an underlying mechanism of CsA-induced nephrotoxicity [31]. The propensity for upregulation of $\mathrm{HO}-1$ protein to occur in renal tubules but not in glomerular cells in 
kidney disease may be related to the differential sensitivity and response to oxidant stress exhibited by these cells. Consistent with the results of previous studies, our results showed that administration of $\mathrm{OA}$ in chronic CsA nephropathy shifted Nrf2 localization from the cytoplasm to the nucleus in kidneys and subsequently activated HO-1 expression compared with the CsA onlytreated group. Unlike previous studies $[17,28,36]$, we did not observe an increase in renal expressions of NQO1, SOD2 and catalase induced by OA in our model of CsA nephropathy. It has been reported that the total cellular balance of pro-oxidants and anti-oxidant intermediates determines how signaling mechanisms respond to various insults [37]. Therefore, the degree of oxidative stress or the innate antioxidant system's response to insults may determine the detailed induction of specific antioxidant genes regulated by Nrf2.

Apoptosis is an essential process in the development and tissue homeostasis of most multicellular organisms, and deregulation of apoptosis has been implicated in the pathogenesis of CsA-induced nephropathy $[38,39]$. CsA induces Bax aggregation and translocation to the mitochondria causing activation of caspase-9, which in turn cleaves and activates caspase-3, an effector caspase, resulting in loss of the mitochondrial transmembrane potential and apoptotic cell death [38]. In the present study, OA administration appeared to protect renal interstitial cells against apoptosis induced by CsA. Notably, the $\mathrm{Bax} / \mathrm{Bcl}-2$ ratio, which corresponds with cell survival in response to apoptotic stimuli, returned to normal levels compared with the control group after administration of OA in CsA-treated mice.

There are some apparent discrepancies in the literature regarding whether the synthetic pentacyclic oleanane derived from OA has anti-apoptotic or pro-apoptotic effects. In an experiment using mouse hepatoma and human hepatopblastoma cells, Nrf2 was shown to control cell apoptosis via upregulation of Bcl-2 transcription and downregulation of $\mathrm{Bax}$ [40]. On the contrary, synthetic pentacyclic oleananes have been reported to induce apoptosis in vitro in human and rodent cancer cells including cells derived from myelomas, leukemia, and sarcomas [27]. These differences can be better understood by considering the specific triterpenoids and cell types studied [27]. Depending on the circumstances, Nrf2 activated by $\mathrm{OA}$ appears to be involved in regulating the balance between anti-apoptotic and pro-apoptotic signaling rather than centralizing the direction of a sole signaling pathway.

There were several interesting aspects of this study in addition to those described above. Although administration of $\mathrm{OA}$ in normal control mice increased the nuclear/total Nrf2 ratio as well as elevated the level of Keap1, the levels of antioxidant enzymes were not significantly altered. This observation suggested that other factors must function in parallel to mediate proper regulation of the downstream targets of Nrf2. In addition, the optimal dose and treatment period of OA for preventing CsA-induced injury has not yet been determined. In our preliminary studies, several additional experiments were performed. First, mice were treated with CsA in the absence or presence of OA for four weeks in order to evaluate its potential as an early or preventative treatment. However, the early treatment schedule was suspended two weeks after starting administration of OA because of significant weight loss and high mortality in mice treated with both CsA and OA. Furthermore, a high dose of OA (50 mg/kg/day) also resulted in significant weight loss and decreased activity in mice 4-5 days after starting administration of OA. Together, these findings suggested that mice do not tolerate high-dose or long-term treatment of $\mathrm{OA}$ in a model of CsA-induced nephropathy.

\section{Conclusions}

In conclusion, the results of the present study demonstrated that delayed administration of OA could provide renoprotective effects against CsA-induced renal injury. The antioxidant potential of OA might be directly correlated with the increased expression of nuclear Nrf2 and subsequently HO-1, which in turn may have surmounted the oxidative stress generated by CsA. In this way, Nrf2 activated by OA may be an important therapeutic target for controlling CsA-induced nephrotoxicity.

\section{Competing interests}

The authors declare that they have no competing interests.

\section{Authors' contributions}

The experiment was designed and implemented by YAH, SJS and SC. Data were analyzed by YAH, JHL, MYK, ENK and ESK. YAH, BSC, CWP and YSC participated in data acquisition and interpretation. $\mathrm{YAH}$ and SC prepared the manuscript. CWP and SC supervised overall project. All authors read and approved the final version of manuscript.

\section{Acknowledgements}

This research was supported by a grant from the Korean Health Technology R\&D Project, Minister of Health and Welfare, Republic of Korea (A111055 to

Cheol Whee Park). The authors wish to thank Hyunmo Jo (Institute of Clinical Medicine Research, The Catholic University of Korea Yeouido St. Mary's Hospital) for his technical assistance.

\section{Author details}

${ }^{1}$ Division of Nephrology, Korea University Guro Hospital, 148, Gurodong-ro, Guro-gu, Seoul 152-703, Republic of Korea. ²Department of Internal Medicine, College of Medicine, The Catholic University of Korea, 222 Banpo-daero, Seoul 137-701, Republic of Korea. ${ }^{3}$ Division of Nephrology, The Catholic University of Korea Yeouido St. Mary's Hospital, 10, 63-ro, Yeongdeungpo-gu, Seoul 150-713, Republic of Korea. ${ }^{4}$ Division of Nephrology, The Catholic University of Korea Incheon St. Mary's Hospital, 56, Dongsu-ro, Bupyeong-gu, Incheon 403-720, Republic of Korea. ${ }^{5}$ Division of Nephrology, The Catholic University of Korea Seoul St. Mary's Hospital, 222, Banpo-daero, Seoul 137-701, Republic of Korea.

Received: 16 December 2013 Accepted: 13 February 2014

Published: 21 February 2014 


\section{References}

1. Myers BD, Ross J, Newton L, Luetscher J, Perlroth M: Cyclosporineassociated chronic nephropathy. N Engl J Med 1984, 311:699-705.

2. Capasso G, Di Gennaro Cl, Della Ragione F, Manna C, Ciarcia R, Florio S, Perna A, Pollastro RM, Damiano S, Mazzoni O, Galletti P, Zappia V: In vivo effect of the natural antioxidant hydroxytyrosol on cyclosporine nephrotoxicity in rats. Nephrol Dial Transplant 2008, 23:1 186-1195.

3. Satyanarayana PS, Chopra K: Oxidative stress-mediated renal dysfunction by cyclosporine A in rats: attenuation by trimetazidine. Ren Fail 2002, 24:259-274.

4. Nguyen T, Nioi P, Pickett CB: The Nrf2-antioxidant response element signaling pathway and its activation by oxidative stress. J Biol Chem 2009, 284:13291-13295.

5. Itoh K, Chiba T, Takahashi S, Ishii T, Igarashi K, Katoh Y, Oyake T, Hayashi N, Satoh K, Hatayama I, Yamamoto M, Nabeshima Y: An Nrf2/small Maf heterodimer mediates the induction of phase II detoxifying enzyme genes through antioxidant response elements. Biochem Biophys Res Commun 1997, 236:313-322.

6. Magesh S, Chen Y, Hu L: Small molecule modulators of Keap1-Nrf2-ARE pathway as potential preventive and therapeutic agents. Med Res Rev 2012, 32:687-726

7. Kobayashi A, Kang MI, Watai Y, Tong Kl, Shibata T, Uchida K, Yamamoto M: Oxidative and electrophilic stresses activate Nrf2 through inhibition of ubiquitination activity of Keap1. Mol Cell Biol 2006, 26:221-229.

8. Abraham NG, Kappas A: Pharmacological and clinical aspects of heme oxygenase. Pharmacol Rev 2008, 60:79-127.

9. Yoh K, Hirayama A, Ishizaki K, Yamada A, Takeuchi M, Yamagishi S, Morito N, Nakano T, Ojima M, Shimohata H, Itoh K, Takahashi S, Yamamoto M: Hyperglycemia induces oxidative and nitrosative stress and increases renal functional impairment in Nrf2-deficient mice. Genes Cells 2008, 13:1159-1170.

10. Yoh K, Itoh K, Enomoto A, Hirayama A, Yamaguchi N, Kobayashi M, Morito N, Koyama A, Yamamoto M, Takahashi S: Nrf2-deficient female mice develop lupus-like autoimmune nephritis. Kidney Int 2001, 60:1343-1353.

11. Liu J: Pharmacology of oleanolic acid and ursolic acid. J Ethnopharmacol 1995, 49:57-68.

12. Patil CR, Jadhav RB, Singh PK, Mundada S, Patil PR: Protective effect of oleanolic acid on gentamicin induced nephrotoxicity in rats. Phytother Res 2010, 24:33-37.

13. Mapanga RF, Tufts MA, Shode FO, Musabayane CT: Renal effects of plant-derived oleanolic acid in streptozotocin-induced diabetic rats. Ren Fail 2009, 31:481-491.

14. Han DH, Piao SG, Song JH, Ghee JY, Hwang HS, Choi BS, Kim J, Yang CW: Effect of sirolimus on calcineurin inhibitor-induced nephrotoxicity using renal expression of KLOTHO, an antiaging gene. Transplantation 2010, 90:135-141.

15. Doh KC, Lim SW, Piao SG, Jin L, Heo SB, Zheng YF, Bae SK, Hwang GH, Min Kl, Chung BH, Yang CW: Ginseng treatment attenuates chronic cyclosporine nephropathy via reducing oxidative stress in an experimental mouse model. Am J Nephrol 2013, 37:421-433.

16. Nataraju A, Saini D, Ramachandran S, Benshoff N, Liu W, Chapman W, Mohanakumar T: Oleanolic Acid, a plant triterpenoid, significantly improves survival and function of islet allograft. Transplantation 2009, 88:987-994

17. Reisman SA, Aleksunes LM, Klaassen CD: Oleanolic acid activates Nrf2 and protects from acetaminophen hepatotoxicity via Nrf2-dependent and Nrf2-independent processes. Biochem Pharmacol 2009, 77:1273-1282.

18. Agarwal R, Vasavada N, Sachs NG, Chase S: Oxidative stress and renal injury with intravenous iron in patients with chronic kidney disease. Kidney Int 2004, 65:2279-2289.

19. Ghosh SS, Massey HD, Krieg R, Fazelbhoy ZA, Ghosh S, Sica DA, Fakhry I, Gehr TW: Curcumin ameliorates renal failure in $5 / 6$ nephrectomized rats: role of inflammation. Am J Physiol Renal Physiol 2009, 296:F1146-F1157.

20. Rodríguez-Iturbe B, Sato T, Quiroz Y, Vaziri ND: AT-1 receptor blockade prevents proteinuria, renal failure, hyperlipidemia, and glomerulosclerosis in the Imai rat. Kidney Int 2004, 66:668-675.

21. Vaziri ND, Bai Y, Ni Z, Quiroz Y, Pandian R, Rodriguez-Iturbe B: Intra-renal angiotensin II/AT1 receptor, oxidative stress, inflammation, and progressive injury in renal mass reduction. J Pharmacol Exp Ther 2007, 323:85-93.
22. Anjaneyulu M, Tirkey N, Chopra K: Attenuation of cyclosporine-induced renal dysfunction by catechin: possible antioxidant mechanism. Ren Fail 2003, 25:691-707.

23. Khan M, Shobha JC, Mohan IK, Rao Naidu MU, Prayag A, Kutala VK: Spirulina attenuates cyclosporine-induced nephrotoxicity in rats. J Appl Toxicol 2006, 26:444-451.

24. Aminzadeh MA, Nicholas SB, Norris KC, Vaziri ND: Role of impaired Nrf2 activation in the pathogenesis of oxidative stress and inflammation in chronic tubulo-interstitial nephropathy. Nephrol Dial Transplant 2013, 28:2038-2045.

25. Kim HJ, Vaziri ND: Contribution of impaired Nrf2-Keap1 pathway to oxidative stress and inflammation in chronic renal failure. Am J Physiol Renal Physiol 2010, 298:F662-F671.

26. Castellano JM, Guinda A, Delgado T, Rada M, Cayuela JA: Biochemical basis of the antidiabetic activity of oleanolic acid and related pentacyclic triterpenes. Diabetes 2013, 62:1791-1799.

27. Liby KT, Sporn MB: Synthetic oleanane triterpenoids: multifunctional drugs with a broad range of applications for prevention and treatment of chronic disease. Pharmacol Rev 2012, 64:972-1003.

28. Wang X, Liu R, Zhang W, Zhang X, Liao N, Wang Z, Li W, Qin X, Hai C: Oleanolic acid improves hepatic insulin resistance via antioxidant, hypolipidemic and anti-inflammatory effects. Mol Cell Endocrinol 2013, 376:70-80.

29. Jung KA, Kwak MK: The Nrf2 system as a potential target for the development of indirect antioxidants. Molecules 2010, 15:7266-7291.

30. Thornalley PJ, Rabbani N: Dietary and synthetic activators of the antistress gene response in treatment of renal disease. J Ren Nutr 2012, 22:195-202.

31. Li J, Jin J, Li M, Guan C, Wang W, Zhu S, Qiu Y, Huang M, Huang Z: Role of $\mathrm{Nrf2}$ in protection against triptolide-induced toxicity in rat kidney cells. Toxicol Lett 2012, 213:194-202.

32. Surh YJ, Kundu JK, Na HK: Nrf2 as a master redox switch in turning on the cellular signaling involved in the induction of cytoprotective genes by some chemopreventive phytochemicals. Planta Med 2008, 74:1526-1539.

33. Farombi EO, Shrotriya S, Na HK, Kim SH, Surh YJ: Curcumin attenuates dimethylnitrosamine-induced liver injury in rats through Nrf2-mediated induction of heme oxygenase-1. Food Chem Toxicol 2008, 46:1279-1287.

34. Morimoto K, Ohta K, Yachie A, Yang Y, Shimizu M, Goto C, Toma T, Kasahara Y, Yokoyama H, Miyata T, Seki H, Koizumi S: Cytoprotective role of heme oxygenase (HO)-1 in human kidney with various renal diseases. Kidney Int 2001, 60:1858-1866.

35. Shimizu M, Ohta K, Yang Y, Nakai A, Toma T, Saikawa Y, Kasahara Y, Yachie A, Yokoyama H, Seki H, Koizumi S: Glomerular proteinuria induces heme oxygenase-1 gene expression within renal epithelial cells. Pediatr Res 2005, 58:666-671.

36. Liu J, Lu YF, Zhang Y, Wu KC, Fan F, Klaassen CD: Oleanolic acid alters bile acid metabolism and produces cholestatic liver injury in mice. Toxicol Appl Pharmacol 2013, 272:816-824.

37. Manna SK, Zhang HJ, Yan T, Oberley LW, Aggarwal BB: Overexpression of manganese superoxide dismutase suppresses tumor necrosis factorinduced apoptosis and activation of nuclear transcription factor-kappaB and activated protein-1. J Biol Chem 1998, 273:13245-13254.

38. Justo P, Lorz C, Sanz A, Egido J, Ortiz A: Intracellular mechanisms of cyclosporin A-induced tubular cell apoptosis. J Am Soc Nephrol 2003, 14:3072-3080.

39. Ortiz A, Lorz C, Catalán M, Ortiz A, Coca S, Egido J: Cyclosporine A induces apoptosis in murine tubular epithelial cells: role of caspases. Kidney Int Supp/ 1998, 68:S25-S29.

40. Niture SK, Jaiswal AK: Nrf2 protein up-regulates antiapoptotic protein Bcl-2 and prevents cellular apoptosis. J Biol Chem 2012, 287:9873-9886.

doi:10.1186/1479-5876-12-50

Cite this article as: Hong et al.: Delayed treatment with oleanolic acid attenuates tubulointerstitial fibrosis in chronic cyclosporine nephropathy through Nrf2/HO-1 signaling. Journal of Translational Medicine 2014 12:50. 